\title{
Editorial
}

\section{Control And Prevention Of Noncommunicable Diseases}

\author{
DOI: https://doi.org/10.3329/kyamcj.v12i2.55434
}

A 'Non-Communicable Disease' (NCD), also known as an 'Aristocratic Disease' or a 'Disease of the Rich' or a 'Lifestyle Disease' is an illness which is not contagious (infectious or communicable) directly from one person to another. ${ }^{1}$ For example Parkinsonism, Autoimmune disorders, Cerebrovascular accidents (CVAs or Strokes), most cardiac diseases, most malignant diseases (cancers), Diabetes, Chronic Kidney Diseases, Osteoarthritis, Osteoporosis, Alzheimer's disease, Cataracts and many other diseases. Non-Communicable Diseases (NCDs) are now emerging as more and more subtle urging global health challenges following more and more avant-grade advancements in treating, controlling and preventing infectious diseases in this era of fourth industrial revolution of human civilization (which has been built upon the third revolution and the digital innovation through the artificial intelligence, the genome editing, the augmented reality, the robotics, the Internet, and the 3-D printing etc.). ${ }^{2}$ NCDs may be acute or chronic. Classically they are not infective, although there are some non-contagious infectious diseases, such as parasitic ones where the parasite's life cycle does not essentially have a direct host-to-host transmission. ${ }^{3}$ NCDs as a whole are now the leading causes of death worldwide, as compared to the Communicable Diseases. The relative mortalities and morbidities are increasing day by day. Historically, many of the NCDs were associated with the economic surge of development and thence were called 'Aristocratic Diseases' or 'Diseases of the Rich'. And many of NCDs are associated with idleness and decreased physical activity in sedentary workers and accordingly these NCDs are also termed as 'Lifestyle Diseases'. ${ }^{4}$ The burden of NDCs in developed, developing and underdeveloped countries has increased albeit, with an estimated over $80 \%$ of the four main types of NCDs: 'cardiovascular diseases (CVDs)', 'malignant diseases (cancers)', 'chronic respiratory diseases(CRDs)' and 'diabetes'. These are now also prevailing in low- and middle-income groups of people. NCDs cause mortalities of about 41 million people every year globally, accounting to about $71 \%$ of all mortalities. ${ }^{5}$ Every year, about 15 million people die from NCDs between the ages of 30 and 69 years; of which over $85 \%$ are described as "premature deaths" prevailing in low- and middle-income groups of people. CVDs per se are incriminated as the causative for most NCD deaths, i.e., 17.9 million people annually, followed by 9.0 million by cancers, 3.9 million by respiratory diseases and 1.6 million by diabetes. ${ }^{5}$ Studies showed that the NCDs paradoxically affecting low- and middle-income groups of people and poor countries cause more than 75 per cent of global NCD deaths, accounting about $32 \mathrm{mil}-$ lion annually. That is to say that deaths from NCDs are on the rise in underdeveloped and developing world. Or otherwise NCDs can no longer be regarded just a burden of only affluent people of the world. If present growth situation of NCDs is continued, by 2020, NCDs will implicate 7 out of every 10 deaths in developing countries, killing 52 million people annually worldwide by 2030.5 Thence WHO (World Health Organization) \& WBHDN (World Bank Human Development Network) have asserted to prioritize treating, controlling and preventing NCDs as a leading 'Global Health Agenda'. ${ }^{6}$

Men, women and children all are susceptible to NCDs. There are such four major risk factors for NCDs as 'tobacco abuse', 'alcohol abuse', 'dietary indiscretion' and 'inadequate physical activities'. Mental illness, behavioral problems, genetic causes \& environmental pollutants have also been identified now as powerful risk factors. In rich societies \& nations, heart diseases and strokes are claiming now less mortalities as compared to that of the past days and to the poor societies and developing nations, as many of the cancer patients are now being cured or are surviving longer and people with diabetes have more adequate opportunities to avail avant-grade health care facilities in rich societies \& nations. In poor societies and poor countries, people with similar illnesses fall sicker more quickly with earlier mortalities and more morbidities. ${ }^{7}$ Smoking, dietary indiscretion, physical inactivity lead to obesity, diabetes and hypertension with their attending complications, morbidities and mortalities. These modifiable risk factors can be manipulated to reduce the risk of many of the NCDs. Likewise we can fight to reduce our exposure to air, soil and water pollutants in our circum-ambient precinct to control and prevent such NCDs as chronic lung diseases. We can avoid unnecessary medication to reduce kidney, liver, blood and mental diseases. ${ }^{8}$ Five important risk factors for non-communicable disease in the top ten leading risks to health are hypertension, hypercholerolemia, tobacco abuse, alcohol abuse, and obesity. The other factors for higher risk of NCDs are economic and social conditions, also known as the "social factors of health". ${ }^{9}$ It has been suggested that if the primary risk factors were eliminated, $80 \%$ of the cases of heart diseases, CVAs and type 2 diabetes and $40 \%$ of cancers 
could have been prevented. Interferences aiming at the main risk factors could have a significant blow in successfully reducing the global burden of NCDs. ${ }^{5}$ Attempts aiming at balanced, better and scientific dietary composition considering the age, sex and other parameters and enhanced physical activity have been verified to treat, control and prevent the NCDs. Thus most of the NCDs are controllable and preventable illnesses, as the most common causes for non-communicable diseases (NCD) include tobacco abuse (smoking), alcohol abuse, poor diets (high consumption of sugar, salt, saturated fats, and trans-fatty acids) and inadequate physical activity. ${ }^{10}$

NCDs are obstacles to poverty alleviation, equities of health and wealth, stability and security of life. Now no family is ascribed as immune to NCDs worldwide. The poverty directly correlates with the vulnerability to NCDs creating extraordinary financial burden to the individual, the family, the society, the health authorities and the state as well. An integrated total approach to physical, mental and spiritual health is the key to healthy life. ${ }^{7}$ Empowerment of community and woman is an essential prerequisite to have healthy life in families concerned. The local, regional and national health authorities will have to highlight NCD activities to implement the required radical changes when needed. Social mobilization, political commitment, mass communication, awareness, active participation of Multi-sectoral multi-stakeholders, volunteers and people altogether can solve the prevailing and the upcoming problems and overcome all obstacles with a view to controlling and preventing NCDs. ${ }^{4}$ There should have policies by policy makers and community leaders to mobilize mass people for successful treatment, control and prevention of these NCDs. Global sustainable measures (GSM) can be executed to halt and eventually even to reverse this emerging threat of NCDs. Potential measures are now being discussed and adopted by the WHO and the FAO (Food and Agriculture Organization) that include reducing the levels of salts in foods, limiting excessive marketing of unhealthy foods and limiting all beverages to children, imposing controls on detrimental alcohol abuse, tobacco abuse and formulating and implementing legislation to limit smoking in public places. Identification, diagnosis, screening and control by most avant-grade treatment protocols for NCDs, as well as palliative care and comprehensive preventive measures, are the keys to the management of NCDs. ${ }^{11}$ Thus the most important way to treat, control and prevent NCDs is to concentrate on reducing the risk factors associated with these diseases. Low-cost solutions are to be formulated by the local, regional and national health authorities and other stakeholders as well to reduce the prevailing modifiable risk factors. Monitoring, surveillance and audit on progress and trends of NCDs and their risk are equally needed for determining policies and priorities. Countries having no and inadequate health insurance coverage are unlikely to have universal access to essential NCD activities. NCD activitiest and interventional management are essential prerequisites for achieving the global target of a $25 \%$ relative reduction in the risk of premature mortality from NCDs by 2025, and the SDGs (Sustainable Development Goals) target of a one-third reduction in premature deaths from NCDs by $2030 .{ }^{5}$ High impact essential NCD management strategies can be provided through a primary health care centres to strengthen early detection and timely treatment of NCDs. Such management strategies are excellent financial investments because, if delivered timely to patients, they can reduce the need for subsequent more expensive management.

Identification, treatment, control and prevention of NCDs are dominant health challenge of the twenty-first century. While it would be unrealistic to expect that all the NCDs can be prevented, a large fraction of them can be controlled and prevented from occurring and prevailing amongst young and middle aged people. Control and prevention of premature mortalities and reduction of NCD-related morbidities and disabilities are the goals that every national health system must pursue and all global partners must corroborate and strictly follow. ${ }^{12}$ These can be achieved as has already been proved in many studies. Control of tobacco abuse is a prime example of how NCD mortality rates had been declined in response to policy and community based initiatives and interventions . Many European countries like Finland and Poland have demonstrated how policies and community-led activities interfering dietary discretion can induce halts and brakes on the CVD-epidemic.

\section{References}

1. NCD Risk Factors Collaboration (NCD-RisC). Worldwide trends in diabetes since 1980: a pooled analysis of 751 population-based studies with 4.4 million participants. The Lancet 2016;387(10027):1513-1530.

2. National, regional and global trends in body-mass index since 1980: systematic analysis of health examination surveys and epidemiological studies with 960 country-years and 9.1 million participants. The Lancet 2011;377(9765):557-567.

3. Lim SS, Vos T, Flaxman AD, Danaei G, Shibuya K, Adair-Rohani H, et al. A comparative risk assessment of burden of disease and injury attributable to 67 risk factors and risk factors clusters in 21 regions, 1990-2010: a systematic analysis for the Global Burden Disease Study 2010. The Lancet 2012;380 (9859):2224-2260. 
4. Kontis V, Mathers CD, Bonita R, Stevens GA, Rehm J, Shield KD, et al. Regional contributions of six preventable risk factors to achieving the $25 ? \times ? 25$ non-communicable disease mortality reduction target: a modelling study. The Lancet Global Health 2015;3(12):e746-e757.

5. GBD 2015 Risk Factors Collaborators. Global, regional, and national comparative risk assessment of 79 behavioural, environmental and occupational, and metabolic risks or clusters of risks, 1990-2015: a systematic analysis for the Global Burden of Disease Study 2015. Lancet, 2016; 388(10053):1659-1724

6. Joffres MR, Campbell NR, Manns B, Tu K. Estimate of the benefits of a population-based reduction in dietary sodium additives on hypertension and its related health care costs in Canada. Canadian Journal of Cardiology 2007;23(6):437-443.

7. Brown IJ, Tzoulaki I, Candeias V, Elliott P. Salt intakes around the world: implications for public health. International Journal of Epidemiology 2009;38(3):791-813.

8. Campbell NRC, Neal BC, MacGregor GA. 2011. Interested in developing a national programme to reduce dietary salt? Journal of Human Hypertension 2011;25:705-710.
9. Marinho de Souza FM, Gawryszewski VP, Orduñez P, Sanhueza A, Espinal MA. Cardiovascular disease mortality in the Americas: current trends and disparities. Heart 2012;98(16):1207-1212.

10. Ferlay J, Soerjomataram I, Dikshit R, Eser S, Mathers C, Rebelo $\mathrm{M}$, et al. Cancer incidence and mortality worldwide: sources, methods and major patterns in GLOBOCAN 2012. International Journal of Cancer 2015;136(5):E359-E386.

11. Goss PE, Lee BL, Badovinac-Crnjevic T, Strasser-Weippl K, Chavarri-Guerra Y, Louis JS, et al. Planning cancer control in Latin America and the Caribbean. The Lancet Oncology 2013;14(5):391-436.

12. NCD Risk Factor Collaboration (NCD-RisC). Trends in adult body-mass index in 200 countries from 1975 to 2014: a pooled analysis of 1698 population-based measurement studies with 19.2 million participants. The Lancet 2016;387(10026):1377-1396.

\section{ABM Moniruddin Professor of Surgery}

